\title{
The Fungal Threat to Global Food Security
}

\section{A foreword article for the Fungal Biology special issue on The Fungal Threat to Food Security}

\author{
Simon V. Avery ${ }^{a, *}$, lan Singleton ${ }^{b}$, Naresh Magan ${ }^{c}$, Gustavo Goldman ${ }^{d}$ \\ Affiliations: \\ aUniversity of Nottingham, School of Life Sciences, University Park, Nottingham NG7 2RD, UK \\ ${ }^{b}$ Edinburgh Napier University, School of Applied Sciences, Sighthill Court, Edinburgh EH11 4BN, UK \\ ${ }^{\circ}$ Cranfield University, Applied Mycology Group, Cranfield MK43 OAL, UK \\ dUniversity of São Paulo, Dept. Ciencias Farmaceut., BR-14040903 Ribeirão Preto, SP, Brazil
}

\section{*Correspondence to Simon.Avery@nottingham.ac.uk}

'Sustainably maintaining a safe and nutritious food supply for an increasing human population is currently one of the key research challenges faced by humanity' and the studies on the impact of fungi on food security is a major part of this research effort. Fungal plant-pathogens destroy up to $30 \%$ of crop products, enough food annually to feed 600 million people, while mycotoxin-producing and food-spoiling fungi further reduce the availability of safe, fresh foods (Medina et al., 2017; Fisher et al., 2018; Maciej et al., 2019). On the other hand, the global market for edible fungi (e.g., mushrooms, mycoprotein) is worth around $€ 38 \mathrm{~B}$ and continues to rise (Willis, 2018; Whittaker et al., 2019). Therefore, different fungi may contribute both to food insecurity and food security (Fig. 1). There is no doubt that effective control of fungi which weakens the security of the food supply is an important socioeconomic goal. This can be driven by targeted research investment to yield fundamental benefits, as population-growth, climate-change and limited land resources will increasingly challenge our capacity to secure adequate food supply in the future; food demand has been projected to increase $>60 \%$ by 2050 (Alexandratos and Bruinsma, 2012). The impacts are not uniform across the globe: food insecurity affects vulnerable, low-income populations the most. The ability of such communities to access food is exacerbated by local population growth, natural resource depletion and land-use conflicts such as dedication of edible-crop cultivation to commercial ventures, e.g., sugar cane for bioethanol production.

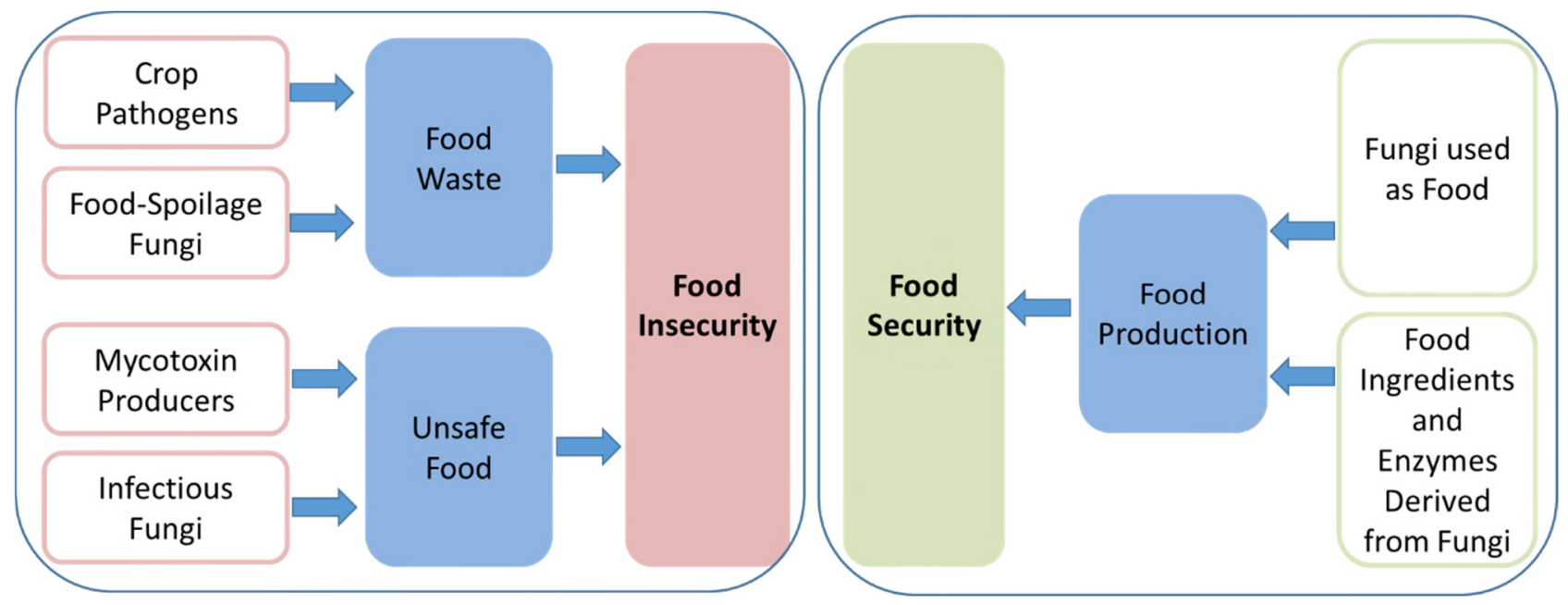

Fig. 1. Different fungal drivers impacting on the level of food (in)security 
Tackling fungal impacts on food waste and safety is likely to depend increasingly on development of innovative solutions (e.g. residue free chemicals) for reducing fungal contamination and growth on food substrates and products. Of key importance is the growth of fungi on stored produce (post harvest) as this can both reduce the nutritional quality of produce that both small scale farmers and larger companies are able to produce and sell and also result in communities being exposed over a longer term to harmful mycotoxins. Indeed, it has now been shown that consumption of aflatoxin contaminated staple foods (class 1a carcinogen), especially maize, results in stunting of infants and children in Lower Middle Income Countries (LMICs; Wild et al., 2015). Thus effective intervention strategies are needed to reduce this level of exposure while conserving food quality and safety. Consumers and the food industry would be the main beneficiaries, where food safety is improved and shelf-life extended. Such research could also inform national and international policies governing food additives, storage and related regulatory guidance for food handling and processing.

With these issues in mind, in 2017-18 the British Council and FAPESP funded an initiative to bring together early career researchers and senior-investigators from the UK and Brazil working in this area. The objective was to challenge and invigorate the new generation of UK and Brazil scientists, through a unified focus on combatting the fungal threat to global food security. A key activity towards achieving this was a three-day Researcher Links workshop, held in September 2018 at the University of São Paulo, Ribeirão Preto campus. The topic of the workshop was "Food security for vulnerable populations: the fungal threat" and involved 33 researcher participants who were funded to attend ( 20 from the UK, 13 from Brazil). A further outcome from this initiative is presented through this Special Issue published in Fungal Biology. The article contributions to this Special Issue all involve researchers who participated in the September 2018 workshop.

Four major, interconnected research strands relevant to fungal impacts on food security were identified by the workshop-consortium (Figure 2). These strands are each active areas of current research, towards which development of new fungal-control measures should be prioritised. For example, the spread of fungicide resistance among crop pathogens is a core concern, as is the robustness of pathogen-resistant crop variants. Fungal metabolism is one element of such resistance where it arises, while diagnosis and monitoring of the incidence of resistance is a crucial element towards effective treatment or introduction of alternative management strategies.

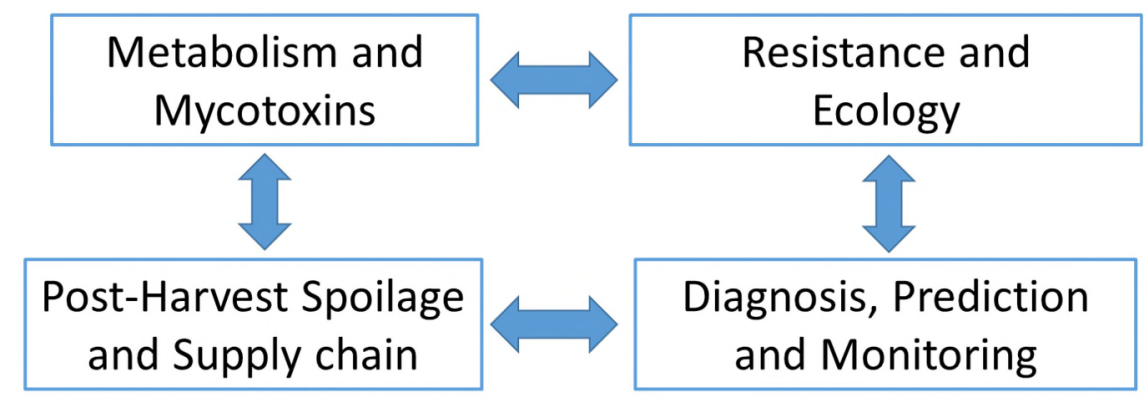

Fig. 2. Four key research strands relevant to fungi and food security. A theme that cross-cuts all these strands is the control of fungal spread and growth.

The selection of papers published in this Special Issue encompasses the research strands described above. Graeme Kettles and Estrella Luna look forward 25 years, to how we may be viewing and handling the fungal threat to food security in 2044, with a particular focus on crop pathogenesis and integrated disease management. Towards a similar aim, Roberta Silva and colleagues build the argument for biological control by fungi of fungal crop pathogens, among other strategies discussed 
in their article. In two articles, Jonas Costa, Taicia Fill and colleagues focus on citrus production - an important crop for the Brazilian economy - and the fungus Penicillium digitatum which causes postharvest citrus decay through a specific host interaction. Secondary metabolites produced by the fungus can be important for the citrus toxicity, but some products may also have potentiallybeneficial insecticidal effects. Louise Mc Namara and colleagues characterise another secondary metabolite, oosporein from Beauveria caledonica, showing that oosporein promotes infection rather than direct killing of insect hosts. This highlights another potential route for beneficial biocontrol by fungi. Other fungal metabolites have toxic effects that are of global concern. Amin Mousavi Khaneghah and colleagues consider the incidence of mycotoxin in coffee-based products, another important export for Brazil and other countries. They highlight positive correlations between mycotoxin incidence and diverse parameters like the level of poverty as well as rainfall. These parameters and others are set to be impacted by climate change in the future. Finally, Carol Verheecke-Vaessen and colleagues examine climate change factors specifically on fungal mycotoxin production and contamination of food substrates (oats). They describe how the incidence of fungal toxins in foods could also be affected by interacting climate change abiotic factors. The forwardlooking content of this and other articles in this Special Issue reflect the likely increasing urgency with which the fungal threat to food security will need to be tackled in the future. The present articles can only touch the tip of the iceberg in addressing all the relevant issues, considering that the issues are wide-ranging and complex. However, these articles do underscore the likelihood of markedly different impacts of the fungal threat to food security in different regions of the world depending partly on whether food is primarily imported or exported, the dependency of the local economy on these markets, the prevalence of poverty and the robustness of supply-chain to underprivileged communities.

\section{Acknowledgements}

This work was supported by the British Council (017-RLWK8-10388) and FAPESP, Brazil (grant number 2017/50201-3).

\section{References}

Alexandratos, N., Bruinsma, J., 2012. World agriculture towards 2030/2050: the 2012 revision. ESA working paper, FAO, Rome.

Fisher, M.C., Hawkins, N.J., Sanglard, D., Gurr, S.J., 2018. Worldwide emergence of resistance to antifungal drugs challenges human health and food security. Science 360, 739-742.

Kaczmarek, M., Avery, S.V., Singleton, I., 2019. Microbes associated with fresh produce: sources, types and methods to reduce spoilage and contamination. Adv. Appl. Microbiol. (in press).

Medina, A., Akbar, A., Baazeem, A., Rodriguez, A., Magan, N., 2017. Climate change, food security and mycotoxins: Do we know enough? Fungal Biol. Rev. 31, 143-154

Whittaker, J.A., Johnson, R.I., Finnigan, T., Avery, S.V., Dyer, P.S., 2019. The biotechnology of Quorn mycoprotein: past, present and future challenges. In: Nevalainen, H. (Ed). Grand Challenges in Fungal Biotechnology. (Series Title: Grand CHalleneges in Biology and Biotechnology). Springer. (In Pres).

Wild, C.P., Miller, J.D. and Groopman, J.D. (2015). Mycotoxin control in Low and Middel Income Countries. IARC Working Group Report 9. International Agency for Research on cancer, 150 cours Thoma, 69372 Lyon Cedex 08, France.

Willis, K.J. (ed), 2018. State of the World's Fungi. Report. Royal Botanic Gardens, Kew: https://stateoftheworldsfungi.org/2018/reports/SOTWFungi_2018_Definition_and_Diversity .pdf 